\title{
Análisis de la legibilidad lingüística de los prospectos de los medicamentos mediante el índice de Flesch-Szigriszt y la escala Inflesz
}

\section{Legibility analysis of drug package leaflets using Flesch-Sszigriszt index and Inflesz score}

\author{
S. Ballesteros-Peña ${ }^{1}$, I. Fernández-Aedo ${ }^{1}$
}

\section{RESUMEN}

Fundamento. Medir y evaluar la legibilidad lingüística de una muestra de prospectos de medicamentos de uso común.

Metodología. Estudio descriptivo, transversal realizado sobre una muestra representativa de prospectos correspondiente a los 30 medicamentos más dispensados en España. Se utilizó el Índice de Flesch-Szigriszt como herramienta de medida y la escala INFLESZ para evaluar la legibilidad lingüística de cada uno de los prospectos. Se consideró que el grado de legibilidad era aceptable cuando el Índice de Flesch-Szigriszt era $\geq 55$.

Resultados. La puntuación media en el Índice de FleschSzigriszt de los prospectos estudiados fue de 52,62 (desviación típica: 4,67), un valor clasificado como "algo difícil" en la Escala INFLESZ. Tan solo 10 de los 30 prospectos presentaba un grado de legibilidad aceptable. Los apartados del prospecto con mayor complejidad gramatical corresponden a "qué es y para qué se utiliza" (indicaciones), "antes de tomar el medicamento" (contraindicaciones) y "efectos adversos".

Conclusiones. Los prospectos de los medicamentos analizados presentan una legibilidad mejorable: las estructuras gramaticales son generalmente complejas, lo que dificulta la comprensión al ciudadano medio. La implicación de todos los profesionales de la salud en la información y resolución de dudas al usuario respecto al consumo de los medicamentos puede resultar imprescindible para un correcto tratamiento.

Palabras clave. Legibilidad. Prospectos de Medicamentos. Comunicación en Salud. Alfabetización en Salud. Farmacia.

\begin{abstract}
Background. To measure and evaluate the legibility of a sample of informative leaflets about common drugs.
\end{abstract}

Methods. A descriptive cross-sectional study was performed, using a representative sample of package leaflets corresponding to the 30 most widely consumed drugs in Spain. The Flesch-Szigriszt Index was used as a measuring tool and the INFLESZ score to evaluate the legibility of leaflets of medicinal products. Documents with a Flesch-Szigriszt Index value $\geq 55$ were considered legible.

Results. The mean Flesch-Szigriszt Index value was 52.62 (standard deviation: 4.67). This score is classified as "a bit difficult" on the INFLESZ score. Only 10 of 30 leaflets obtained an acceptable level of legibility. The sections of the leaflets with higher grammatical complexity were "what it is and what it is used for" (indications), "before taking the medication" (contraindications) and "adverse effects".

Conclusions. The legibility of the package leaflets analyzed could be improved: grammatical structures are usually convoluted, and their comprehension by ordinary citizens belonging to the general public is difficult. The involvement of health care professionals in resolving doubts and giving information to users about the consumption of drugs may be essential for a correct treatment.

Key words. Legibility. Medicine Package Inserts. Health Communication. Health Literacy. Pharmacy.
1. Escuela Universitaria de Enfermería. Departamento de Enfermería I. Universidad del País Vasco-Euskal Herriko Unibertsitatea.
Recepción: 15 de septiembre de 2013

Aceptación provisional: 15 de octubre de 2013

Aceptación definitiva: 17 de octubre de 2013

\section{Correspondencia:}

Sendoa Ballesteros Peña

Universidad del País Vasco/

Euskal Herriko Unibertsitatea

Escuela Universitaria de Enfermería

Departamento de Enfermería I (Campus de Leioa)

$\mathrm{B}^{\mathrm{o}}$ Sarriena $\mathrm{s} / \mathrm{n}$

48940 Leioa (Bizkaia)

sendoa.ballesteros@ehu.es 


\section{INTRODUCCIÓN}

En español solo existe un vocablo para traducir dos términos ingleses, "readability" y "legibility": "legibilidad". Aunque desde el punto de vista semántico estos términos son sinónimos, el uso les ha otorgado significados diferentes.

La legibilidad tipográfica ("readability") tiene que ver con la forma de presentar los escritos para que puedan leerse sin dificultad, es decir, la cualidad de un texto de ser fácilmente leído desde un punto de vista mecánico. Éste parámetro valora, entre otros, el tipo, color y tamaño de la letra o del papel, la presencia o ausencia de figuras o la maquetación.

La legibilidad lingüística ("legibility", también denominada comprensibilidad, perspicuidad o lecturabilidad) se refiere a la facilidad que ofrecen los textos para ser comprendidos sin necesidad de realizar grandes esfuerzos. Un texto puede ser más o menos fácil de comprender en función de la complejidad gramatical de la construcción del mensaje (tamaño y número de palabras y frases, promedio de sílabas por palabra, palabras por frase...) y de factores personales del lector (nivel cultural e intelectual, interés por el tema $)^{1}$.

Existe una notable diferencia entre legibilidad tipográfica y lingüística. Mientras que la primera se refiere a la facilidad con la que los lectores pueden decodificar la información en un documento, la segunda hace referencia al contenido del mismo. Sin embargo, ambas tienen un efecto importante sobre el éxito o fracaso de la lectura de un documento.

Diversos autores han constatado la dificultad de lectura y compresión de los prospectos de los medicamentos y la necesidad de mejorar su contenido ${ }^{2}$. En este sentido un estudio realizado en el año 2008 indicó que solo 5 prospectos de los 30 analizados podían ser considerados legibles ${ }^{3}$. Otro aspecto a tener en cuenta es que los prospectos no invitan a su lectura, ya que estos contienen demasiada información y el lenguaje puede resultar demasiado técnico o científico ${ }^{4}$.
El prospecto de un medicamento es un texto informativo que se ha de dirigir a los pacientes/usuarios y no a los profesionales del ámbito sanitario. El objetivo principal del prospecto es garantizar un uso correcto del medicamento, asegurando la eficacia y seguridad de los mismos. Una deficiente comprensión de la información dispuesta en los prospectos puede traducirse en un incremento del riesgo de iatrogenia para el consumidor, que además de generar consecuencias de gravedad variable para su salud, ocasionaría un coste económico importante para los servicios sanitarios ${ }^{5}$. Y es que, los ingresos realizados en los servicios de urgencias en España a consecuencia de una mala administración de medicamentos es un hecho, tal y como se constata en diversos estudios ${ }^{6-8}$.

Aunque el punto de vista de los profesionales sanitarios revele que el prospecto se considera de gran utilidad para los pacientes y se enfatice que el texto ha de ser inteligible por una población con conocimientos básicos o generales, los pacientes no están satisfechos con la información que aparece en los prospectos $^{4}$. De hecho, en opinión del presidente del Foro Español de Pacientes, Albert Jovell, el lenguaje de los prospectos resulta demasiado complicado, perjudicando notablemente las posibilidades de que los pacientes lleven a cabo correctamente su tratamiento ${ }^{9}$.

A partir de la necesidad de determinar si la información de un texto es eficaz para comunicar un mensaje a los usuarios y consumidores se han creado diferentes instrumentos que ayudan a determinar adecuadamente el grado de comprensibilidad de un texto para determinados grupos de lectores.

La legibilidad lingüística ha sido objeto de estudio de una forma más amplia porque es tributaria de ser analizada aplicando técnicas de medición más objetivas (cuantitativas). Los análisis de legibilidad lingüística emplean fórmulas matemáticas para evaluar la dificultad de comprensión, partiendo del principio de que un texto es más complicado cuanto más compleja es su construcción gramatical. 
Existen múltiples sistemas y fórmulas para medir la legibilidad lingüística de los textos $^{10}$ (como los índices de Flesch, Fernández-Huerta, LEGIN o Gunning-Fog, entre otros), si bien, ninguna ha sido concebida específicamente para el ámbito sanitario. No obstante, estas herramientas permiten una estimación de la dificultad técnica que pueden presentar los materiales escritos utilizados en el entorno sociosanitario y, de manera indirecta, también valorar su aplicabilidad.

Es razonable pensar que el éxito de un tratamiento farmacológico no sólo va a radicar en la eficacia del fármaco, sino también en la correcta indicación e información que recibe el paciente sobre su uso y consumo, ya sea por parte de los profesionales sanitarios o por medios indirectos como el prospecto del medicamento. Por ello el objetivo de este trabajo se ha centrado en medir y evaluar la legibilidad lingüística de una muestra representativa de prospectos de medicamentos de uso común en nuestro entorno.

\section{METODOLOGÍA}

Se diseñó un estudio descriptivo y transversal donde se analizaron los prospectos de los 30 medicamentos con mayor número de envases dispensados en España entre noviembre 2006 y octubre 2007 (Tabla 1), al ser éstos los últimos datos facilitados por la consultora IMS Health ${ }^{11}$. No se incluyeron los fármacos que sólo disponían de presentaciones de uso tópico, en inhalador o en gotas. Los criterios de muestreo empleados atienden al interés práctico de evaluar los medicamentos más consumidos por los ciudadanos españoles.

La obtención de los prospectos para cada medicamento se realizó en junio de 2013 a través de la página web de la Agen- cia Española de Medicamentos y Productos Sanitarios (en formato de Adobe Acrobat). Cuando de un mismo medicamento existía más de una presentación comercial, se optó por el prospecto de la más frecuentemente dispensada.

Posteriormente, cada prospecto se transformó a un archivo de texto sin formato y se corrigieron defectos gramaticales (se insertaron signos de puntuación ausentes y se eliminaron espacios innecesarios entre palabras), se suprimieron las tablas, cuadros y guiones o viñetas, las abreviaturas fueron sustituidas por sus términos originales y los caracteres que expresaban ideas fueron sustituidos por la forma gramatical tradicional (por ejemplo, "mg/día" por "miligramos al día"). También se eliminaron los datos de composición del medicamento e información adicional como la titularidad del laboratorio responsable de la fabricación y comercialización.

Finalmente, se analizó el grado de legibilidad para los siguientes contenidos de cada prospecto: indicaciones ("qué es y para qué se utiliza"); contraindicaciones, precauciones e interacciones ("antes de tomar el medicamento"); posología (“cómo tomar el medicamento"); efectos adversos y conservación.

Para calcular la legibilidad lingüística de los textos seleccionados se utilizó la aplicación informática INFLESZ 1.0, que emplea la versión española validada del Îndice de Flesch, la Fórmula de Perspicuidad o Índice de Flesch-Szigriszt (IFSZ) ${ }^{12}$, al ser considerado de referencia en el momento actual para el análisis de la dificultad sintáctica de los textos en lengua española ${ }^{13}, \mathrm{y}$ la escala INFLESZ ${ }^{3}$, una herramienta de evaluación de resultados que establece una asociación entre el IFSZ y el nivel de dificultad de lectura (Tabla 2). La Escala INFLESZ determina que la comprensibilidad de un texto es aceptable cuando el IFSZ $\geq 55$. 
Tabla 1: Listado de medicamentos con más envases dispensados en España entre noviembre 2006 y octubre 2007 (Fuente: IMS Health)

\begin{tabular}{|c|c|c|c|}
\hline & Nombre comercial & Principios activos & Envases dispensados \\
\hline 1 & Nolotil & Metamizol magnésico & 14.943 .764 \\
\hline 2 & Efferalgan & Paracetamol & 13.784 .387 \\
\hline 3 & Gelocatil & Paracetamol & 13.042 .299 \\
\hline 4 & Adiro & Ácido acetilsalicílico & 12.716 .646 \\
\hline 5 & Augmentine & Amoxicilina trihidrato, Clavulanato de potasio & 10.617 .537 \\
\hline 6 & Orfidal Wyeth & Lorazepam & 8.939 .766 \\
\hline 7 & Neobrufen & Ibuprofeno & 8.491 .256 \\
\hline 8 & Dianben & Metformina hidrocloruro & 8.463 .688 \\
\hline 9 & Termalgin & Paracetamol & 8.250 .873 \\
\hline 10 & Lexatin & Bromazepam & 8.119 .214 \\
\hline 11 & Almax & Almagato & 8.100 .143 \\
\hline 12 & Trankimazin & Alprazolam & 7.601 .773 \\
\hline 13 & Flumil & Acetilcisteína & 7.333 .923 \\
\hline 14 & Sintrom & Acenocumarol & 6.188 .246 \\
\hline 15 & Frenadol & $\begin{array}{l}\text { Paracetamol, Clorfenamina maleato, } \\
\text { Dextrometorfano hidrobromuro }\end{array}$ & 6.044 .661 \\
\hline 16 & Aspirina & Ácido acetilsalicílico & 6.039 .535 \\
\hline 17 & Dalsy & Ibuprofeno & 5.724 .879 \\
\hline 18 & Espidifen & Ibuprofeno arginato & 5.336 .522 \\
\hline 19 & Tranxilium & Clorazepato de dipotasio & 5.320 .873 \\
\hline 20 & Omeprazol Ratiopharm EFG & Omeprazol & 5.319 .182 \\
\hline 21 & Cardyl & Atorvastatina cálcica trihidrato & 5.092 .508 \\
\hline 22 & Yasmín & Drospirenona, etinilestradiol & 5.007 .833 \\
\hline 23 & Seguril & Furosemida & 4.820 .091 \\
\hline 24 & Omeprazol Cinfamed EFG & Omeprazol & 4.626 .334 \\
\hline 25 & Flutox & Cloperastina fendizoato & 4.519 .717 \\
\hline 26 & Zarator & Atorvastatina calcio trihidrato & 4.500 .900 \\
\hline 24 & A.A.S. & Ácido acetilsalicílico & 4.427 .621 \\
\hline 28 & Plantaben & Plantago ovata & 4.298 .783 \\
\hline 29 & Noctamid & Lormetazepam & 4.043 .852 \\
\hline 30 & Enantyum & Dexketoprofeno trometamol & 4.031 .676 \\
\hline
\end{tabular}

EFG: Especialidad Farmacéutica Genérica.

Tabla 2. Relación entre el Índice de Flesch-Szigriszt y el grado de dificultad de la legibilidad en la Escala INFLESZ

\begin{tabular}{ccl}
\hline $\begin{array}{c}\text { Índice de } \\
\text { Flesch-Szigriszt }\end{array}$ & $\begin{array}{c}\text { Grado dificultad } \\
\text { INFLESZ }\end{array}$ & \multicolumn{1}{c}{$\begin{array}{c}\text { Nivel de estudios preciso } \\
\text { (y tipos de publicación) }\end{array}$} \\
\hline$<40$ & Muy difícil & Universitario (textos universitarios o científicos) \\
\hline $40-54$ & Algo difícil & $\begin{array}{l}\text { Educación media superior, bachillerato (textos de divulgación } \\
\text { científica, prensa especializada) }\end{array}$ \\
\hline $55-64$ & Normal & Educación secundaria (prensa general o deportiva) \\
\hline $65-79$ & Bastante fácil & Educación primaria ("best sellers", prensa del corazón) \\
$\geq 80$ & Muy fácil & Educación primaria (historietas, cómics) \\
\hline
\end{tabular}


Se calcularon las medias y desviaciones típicas (Dt) de las puntuaciones del IFSZ pero, dado el limitado número de casos estudiados, no se aplicaron pruebas estadísticas para la comparación de medias.

\section{RESULTADOS}

La puntuación media en el IFSZ de los prospectos analizados fue de 52,62 (Dt: $4,67)$, un valor clasificado como "algo difícil" en la Escala INFLESZ. En la tabla 3 se recogen las puntuaciones del IFSZ de cada prospecto estudiado y de cada uno de sus apartados.

Tabla 3. Prospectos estudiados, índices de legibilidad y grado de dificultad

\begin{tabular}{|c|c|c|c|c|c|c|c|}
\hline \multirow[t]{2}{*}{$\begin{array}{l}\text { Nombre comercial y } \\
\text { presentación }\end{array}$} & \multirow[t]{2}{*}{$\begin{array}{c}\text { Fecha revisión } \\
\text { prospecto }\end{array}$} & \multicolumn{6}{|c|}{$\begin{array}{l}\text { Índice Flesch-Szigriszt } \\
\text { (Grado INFLESZ) }\end{array}$} \\
\hline & & 1 & 2 & 3 & 4 & 5 & Global \\
\hline A.A.S. $500 \mathrm{mg}$ comp. & Marzo 2013 & $61,91(\mathrm{~N})$ & $49,29(\mathrm{AD})$ & $62,56(\mathrm{~N})$ & $53,89(\mathrm{AD})$ & $74,27(\mathrm{BF})$ & $55,09(\mathrm{~N})$ \\
\hline Adiro 100 mg comp. & Noviembre 2012 & $45,75(\mathrm{AD})$ & $40,74(\mathrm{AD})$ & $46,67(\mathrm{AD})$ & 40,91 (AD) & $66,92(\mathrm{BF})$ & $44,06(\mathrm{AD})$ \\
\hline Almax comp. masticables & Agosto 2010 & $46,69(\mathrm{AD})$ & $49,87(\mathrm{AD})$ & $58,26(\mathrm{~N})$ & $56,07(\mathrm{~N})$ & $61,26(\mathrm{~N})$ & $52,28(\mathrm{AD})$ \\
\hline Aspirina 500 mg comp. & Febrero 2013 & 35,29 (MD) & $42,71(\mathrm{AD})$ & $49,79(\mathrm{AD})$ & $48,71(\mathrm{AD})$ & $64,73(\mathrm{~N})$ & $46,51(\mathrm{AD})$ \\
\hline Augmentine 500/125mg comp. & Febrero 2010 & $41,81(\mathrm{AD})$ & $53,76(\mathrm{AD})$ & $62,71(\mathrm{~N})$ & $58,86(\mathrm{~N})$ & $67,12(\mathrm{BF})$ & $57,54(\mathrm{~N})$ \\
\hline Cardyl 10 mg comp. & Marzo 2011 & $56,43(\mathrm{~N})$ & $51,51(\mathrm{AD})$ & 72,49 (BF) & $62,77(\mathrm{~N})$ & $65,23(\mathrm{~N})$ & $57,35(\mathrm{~N})$ \\
\hline Dalsy $40 \mathrm{mg} / \mathrm{ml}$ suspensión or al & Junio 2010 & 37,41 (MD) & $46,04(\mathrm{AD})$ & $52,70(\mathrm{AD})$ & $51,76(\mathrm{AD})$ & $66,62(\mathrm{BF})$ & $50,48(\mathrm{AD})$ \\
\hline Dianben $850 \mathrm{mg}$ comp. & Octubre 2010 & $61,76(\mathrm{~N})$ & $55,17(\mathrm{~N})$ & $64,64(\mathrm{~N})$ & $59,50(\mathrm{~N})$ & 67,97 (BF) & $59,69(\mathrm{~N})$ \\
\hline Efferalgan 1g comp. eferv. & Abril 2002 & 32,85 (MD) & $49,01(\mathrm{AD})$ & $61,30(\mathrm{~N})$ & $42,13(\mathrm{AD})$ & $58,12(\mathrm{~N})$ & $51,83(\mathrm{AD})$ \\
\hline Enantyum 12,5 mg comp. & Julio 2012 & $40,00(\mathrm{MD})$ & $44,70(\mathrm{AD})$ & $61,12(\mathrm{~N})$ & $46,93(\mathrm{AD})$ & $79,32(\mathrm{BF})$ & $49,56(\mathrm{AD})$ \\
\hline Espidifen $400 \mathrm{mg}$ granulado & Julio 2011 & $45,03(\mathrm{AD})$ & $46,82(\mathrm{AD})$ & $56,33(\mathrm{~N})$ & $51,42(\mathrm{AD})$ & 67,18 (BF) & $51,24(\mathrm{AD})$ \\
\hline Flumil $200 \mathrm{mg}$ granulado & & $36,95(\mathrm{MD})$ & $51,79(\mathrm{AD})$ & $51,91(\mathrm{AD})$ & 37,91 (MD) & $62,55(\mathrm{~N})$ & $50,03(\mathrm{AD})$ \\
\hline Flutox $3,54 \mathrm{mg} / \mathrm{ml}$ jarabe & Abril 2013 & $68,93(\mathrm{BF})$ & $61,79(\mathrm{~N})$ & $56,83(\mathrm{~N})$ & $65,90(\mathrm{BF})$ & $69,21(\mathrm{BF})$ & $60,77(\mathrm{~N})$ \\
\hline Frenadol comp. eferv. & Julio 2003 & $43,07(\mathrm{AD})$ & $46,96(\mathrm{AD})$ & $55,03(\mathrm{~N})$ & $35,31(\mathrm{MD})$ & $40,49(\mathrm{AD})$ & $47,53(\mathrm{AD})$ \\
\hline Gelocatil 650 mg comp. & Agosto 2010 & $58,54(\mathrm{AD})$ & $42,87(\mathrm{AD})$ & $57,71(\mathrm{~N})$ & $59,24(\mathrm{~N})$ & $63,10(\mathrm{~N})$ & $52,06(\mathrm{AD})$ \\
\hline Orfidal Wyeth 1 mg comp. & Septiembre 2009 & $45,06(\mathrm{AD})$ & $42,24(\mathrm{AD})$ & $44,54(\mathrm{AD})$ & $48,64(\mathrm{AD})$ & $69,82(\mathrm{BF})$ & $46,33(\mathrm{AD})$ \\
\hline Lexatin 1,5 mg cáps. & Junio 2008 & $59,40(\mathrm{~N})$ & $58,96(\mathrm{~N})$ & $62,33(\mathrm{~N})$ & $49,56(\mathrm{AD})$ & 77,24 (BF) & $58,51(\mathrm{~N})$ \\
\hline Neobrufen 600 mg comp. & Septiembre 2012 & $40,09(\mathrm{AD})$ & $45,14(\mathrm{AD})$ & $54,70(\mathrm{AD})$ & $50,83(\mathrm{AD})$ & $62,94(\mathrm{~N})$ & $49,64(\mathrm{AD})$ \\
\hline Noctamid 1 mg comp. & Septiembre 2012 & $59,45(\mathrm{~N})$ & $44,45(\mathrm{AD})$ & $56,68(\mathrm{~N})$ & $42,69(\mathrm{AD})$ & $69,72(\mathrm{BF})$ & $47,54(\mathrm{AD})$ \\
\hline Nolotil 575 mg cáps. & Mayo 2011 & $44,10(\mathrm{AD})$ & $51,36(\mathrm{AD})$ & $51,15(\mathrm{AD})$ & $55,46(\mathrm{~N})$ & 67,93 (BF) & $53,52(\mathrm{AD})$ \\
\hline Omeprazol Cinfamed EFG cáps. & Agosto 2012 & $37,52(\mathrm{MD})$ & $51,23(\mathrm{AD})$ & $56,09(\mathrm{~N})$ & $53,63(8 \mathrm{AD})$ & $68,32(\mathrm{BF})$ & $53,40(\mathrm{AD})$ \\
\hline Omeprazol Ratiopharm EFG cáps. & Febrero 2013 & $51,38(\mathrm{AD})$ & $47,62(\mathrm{AD})$ & $61,50(\mathrm{~N})$ & $57,45(\mathrm{~N})$ & $64,08(\mathrm{~N})$ & $56,38(\mathrm{~N})$ \\
\hline Plantaben 3,5 g polvo eferv. & Enero 2012 & $53,43(\mathrm{AD})$ & $50,57(\mathrm{AD})$ & $57,69(\mathrm{AD})$ & $53,10(\mathrm{AD})$ & 74,95 (BF) & $54,80(\mathrm{AD})$ \\
\hline Seguril 40 mg comp. & Mayo 2010 & $4,45(\mathrm{AD})$ & $43,32(\mathrm{AD})$ & $54,9(\mathrm{AD})$ & 36,11 (MD) & 74,60 (BF) & $44,91(\mathrm{AD})$ \\
\hline Sintrom $4 \mathrm{mg}$ comp & Septiembre 2011 & $45,91(\mathrm{AD})$ & $43,31(\mathrm{AD})$ & $63,01(\mathrm{~N})$ & $64,52(\mathrm{~N})$ & 70,04 (BF) & $53,38(\mathrm{AD})$ \\
\hline Termalgin 500 mg comp. & Octubre 2012 & 71,74 (BF) & $46,76(\mathrm{AD})$ & $65,24(\mathrm{BF})$ & $51,80(\mathrm{AD})$ & 71,85 (BF) & $57,28(\mathrm{~N})$ \\
\hline Trankimazin 1 mg comp. & Julio 2012 & $45,76(\mathrm{AD})$ & $44,07(\mathrm{AD})$ & $53,00(\mathrm{AD})$ & 38,10 (MD) & $68,88(\mathrm{BF})$ & $47,08(\mathrm{AD})$ \\
\hline Tranxilium 5 mg cáps. & Junio 2011 & $44,08(\mathrm{AD})$ & $52,31(\mathrm{AD})$ & $54,56(\mathrm{AD})$ & 38,31 (MD) & 72,59 (BF) & $52,63(\mathrm{AD})$ \\
\hline Yasmín 3/0,03 mg comp. & Abril 2012 & 37,24 (MD) & $57,92(\mathrm{~N})$ & $65,42(\mathrm{BF})$ & $52,81(\mathrm{AD})$ & 76,39 (BF) & $61,11(\mathrm{~N})$ \\
\hline Zarator $20 \mathrm{mg}$ comp & Junio 2012 & $53,87(\mathrm{AD})$ & $44,19(\mathrm{AD})$ & 66,20 (BF) & $63,10(\mathrm{~N})$ & 68,88 (BF) & $56,02(\mathrm{~N})$ \\
\hline $\begin{array}{c}\text { Puntuaciones } \\
\text { Media } \pm \mathrm{Dt}\end{array}$ & & $\begin{array}{l}46,86 \pm 12,73 \\
(\mathrm{AD})\end{array}$ & $\begin{array}{l}48,55 \pm 5,22 \\
(\mathrm{AD})\end{array}$ & $\begin{array}{c}57,90 \pm 6,09 \\
(\mathrm{~N})\end{array}$ & $\begin{array}{l}50,91 \pm 8,66 \\
\text { (AD) }\end{array}$ & $\begin{array}{c}67,74 \pm 7,02 \\
\text { (BF) }\end{array}$ & $\begin{array}{c}52,62 \pm 4,67 \\
\text { (AD) }\end{array}$ \\
\hline
\end{tabular}

cáps: cápsulas; comp,: comprimidos; Dt: desviación típica; eferv: efervescente; EFG: Especialidad Farmacéutica Genérica; (BF): bastante fácil; (N): normal; (AD): algo difícil; (MD): muy difícil.

Apartados: 1: qué es y para qué se utiliza (indicaciones); 2: antes de tomar el medicamento (contraindicaciones); 3: cómo tomar el medicamento (posología); 4: efectos adversos; 5: conservación. 
Tan sólo 10 de los 30 medicamentos presentaba prospectos con puntuaciones del IFSZ superiores a 55 (Fig. 1). Además hay que destacar que de estos 10 prospectos 8 son prospectos revisados en el año
2010 o en años posteriores. De hecho, el medicamento Flutox® que obtiene la mayor puntuación global en el IFSZ, fue revisado en el año 2013.

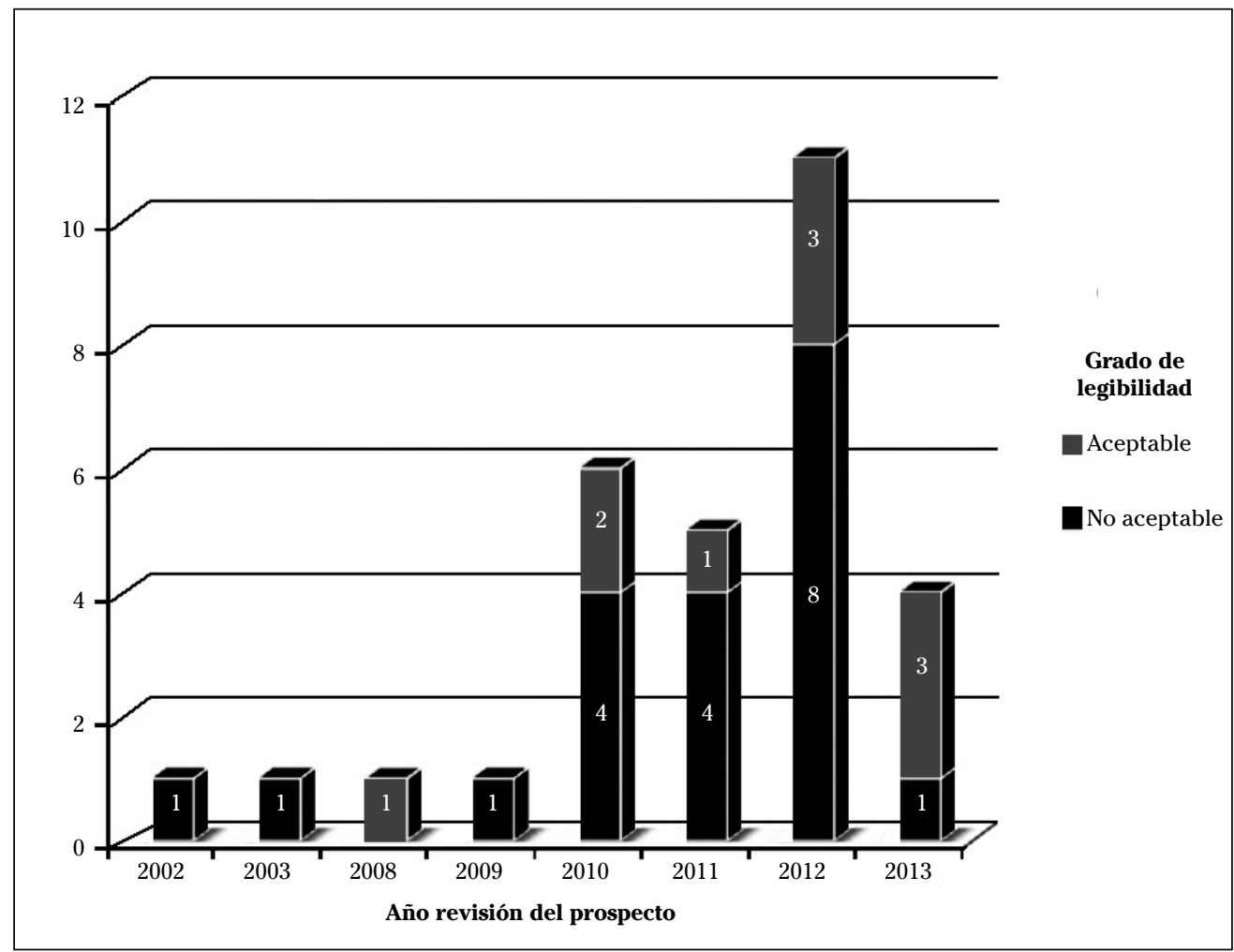

Aceptable: Índice de Flesch-Szigriszt $\geq 55$ (Grado INFLESZ: Normal, Bastante fácil o Muy fácil). No aceptable: Índice de Flesch-Szigriszt < 55 (Grado INFLESZ: Algo difícil o Muy difícil).

Figura 1. Grado de legibilidad de los prospectos estudiados en función del año de revisión del texto

En la figura 2 se representan los grados de dificultad de legibilidad según la Escala INFLESZ para cada apartado de los prospectos analizados. Es posible observar que el apartado con menor dificultad de legibilidad es el que corresponde a la conservación, ya que emplea construcciones gramaticales poco complejas. 


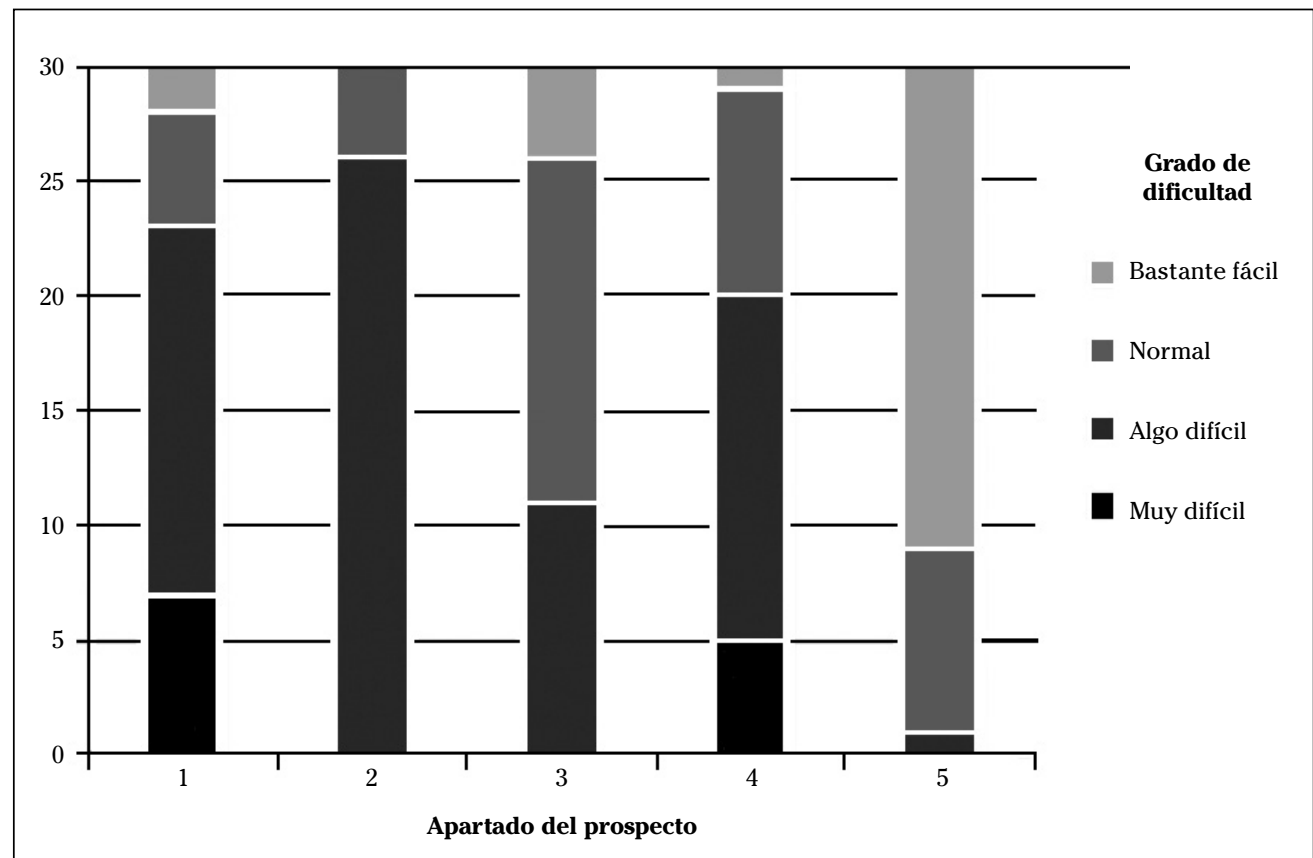

1: qué es y para qué se utiliza; 2: antes de tomar el medicamento; 3: cómo tomar el medicamento; 4: efectos adversos; 5: conservación.

Figura 2. Clasificación de la dificultad de legibilidad según la Escala INFLESZ para cada apartado de los prospectos analizados

\section{DISCUSIÓN}

Son abundantes los trabajos que se han centrado en analizar la legibilidad lingüística de diversos materiales escritos en lengua española en el ámbito de la salud, como documentos de consentimiento informado ${ }^{14}$, textos de educación para la salud ${ }^{15} \mathrm{y}$, en menor medida, prospectos de medicamentos ${ }^{16}$. Así, fruto del trabajo de un Comité de Lectura de Prospectos creado en el año 2001 y formado por expertos independientes, se lograron elaborar en el año 2007 unas directrices iniciales que compaginan el rigor y la comprensibilidad de la información sobre los medicamentos dirigida a los consumidores ${ }^{2}$.

El hecho de existir recomendaciones ${ }^{17} \mathrm{y}$ normativas $^{18-19}$ que regulan los criterios de legibilidad de los prospectos de los medicamentos antes de que sean comercializados puede haber disuadido a los investigadores de abordar este aspecto, lo que podría explicar la escasa literatura al respecto. No obstante, es preciso tener en cuenta que las recomendaciones referidas en la normativa anterior se centran particularmente en aspectos de carácter tipográfico, aunque también ofrece pautas básicas de construcción sintáctica y semántica de los textos. Además, no incluye otras metodologías de evaluación de la legibilidad más allá de las técnicas cualitativas de consulta a pacientes y consumidores ${ }^{20}$.

La aplicación de índices cuantitativos en la evaluación de la legibilidad lingüística ofrece la oportunidad de realizar comparaciones objetivas entre distintos prospectos o clasificar los textos en función del nivel educativo requerido para la correcta compresión de su contenido.

Siguiendo esta metodología, puede llegarse a la conclusión de que la gran mayoría de los prospectos analizados en este trabajo no consiguen un índice de legibilidad aceptable, ya que tienden a emplear 
construcciones sintácticas complejas, con palabras y frases excesivamente largas que exigen habilidades lectoras superiores a las de un ciudadano medio. No obstante, sí se aprecia una mejora de la legibilidad en los prospectos revisados a partir del año 2010, tal vez como respuesta a las demandas reiterativas por parte de las organizaciones sanitarias y de pacientes. Curioso resulta el caso del Gelocatil ${ }^{\circledR}$ y la Aspirina ${ }^{\circledR}$, ya que tratándose de medicamentos de venta libre y cuyos prospectos están revisados en los años 2010 y 2013 respectivamente, no consiguen un índice de legibilidad con grado de dificultad "normal". En concordancia con estos datos se encuentra el estudio realizado en medicamentos de venta libre cuyo principio activo es el paracetamol ${ }^{21}$.

Es preciso tener en cuenta que los receptores de la información de los prospectos con frecuencia son personas con nivel cultural y con herramientas de compresión dispares, cuyo único denominador común radica en la necesidad de información sobre el fármaco ${ }^{4}$. Cabría suponer que las personas que tienen un alfabetismo en salud más bajo (es decir, una menor capacidad de obtener, procesar y entender la información básica de salud para tomar decisiones apropiadas para su autocuidado) son los adultos mayores, las minorías raciales y étnicas, las personas con bajo nivel educativo, con escasos ingresos económicos y/o con compromiso del estado de salud ${ }^{22} \mathrm{y}$, por ende, representan el estrato más vulnerable ante los riesgos derivados del déficit de información ${ }^{23}$. En este sentido, España presenta unos indicadores poco halagüeños, que podrían verse magnificados a corto plazo como consecuencia de la crisis económica actual: en el año 2011 el 46\% de la población adulta no había alcanzado un nivel de estudios superior a la primera etapa de la Educación Secundaria, frente al $24 \%$ de media de los países de la Unión Europea ${ }^{24}$ y la tasa de desempleo crece estrepitosamente hasta superar el $26 \%$ en 2013, situándose, tras Grecia, en el segundo país con mayor tasa de paro de la zona euro.

Además, las pocas herramientas en alfabetismo en salud son un obstáculo significativo para poder acceder completamente a un cuidado médico efectivo ${ }^{25}$. Las preferencias de los pacientes, sin una completa comprensión de las opciones de tratamiento, pueden ser por lo tanto una fuente de disparidad racial y étnica en el cuidado $^{26}$. Muchas veces el rechazo de tratamiento y, más comúnmente, la poca adherencia a él son el resultado de una falta de conocimiento, malas experiencias previas en el sistema de salud y falta de comprensión ${ }^{26}$, y desde los servicios socio sanitarios se debería intervenir para elaborar y/o aplicar estrategias apropiadas con que solventar esta deficiencia, en pos de aumentar el empoderamiento del paciente en la toma de decisiones referentes a su salud. El alfabetismo en salud en un importante componente para mejorar la calidad del cuidado $^{22,27}$ ] y mejorar su efectividad ${ }^{28}$ y en ésta línea de acción, son ya varios los materiales existentes en nuestro entorno diseñados para facilitar la comprensión de diversas actividades de autocuidado ${ }^{29,30}$.

Este estudio presenta algunas limitaciones que merecen ser consideradas. La más importante proviene del instrumento utilizado para la medición de la legibilidad de los textos, puesto a día de hoy aún no se ha establecido con detalle la fiabilidad de los análisis de legibilidad, habiéndose objetivado cierto grado de variabilidad en los resultados tras la aplicación de diferentes índices ${ }^{3}$. Es preciso tener en cuenta que aunque el uso de los índices de legibilidad lingüística está muy extendido, su valor es meramente orientativo y no constituye un método de evaluación exacto.

La segunda limitación estriba del carácter dinámico y de los procesos de revisión continúa al que se encuentran sometidos los prospectos de los medicamentos analizados, que pueden comprometer la generalización de los resultados obtenidos de cara a un futuro próximo, dado el planteamiento transversal de éste estudio.

Finalmente, y a tenor de los datos obtenidos en este trabajo, se constata que los textos de los prospectos de los medicamentos analizados presentan estructuras gramaticalmente complejas, que se traduce en una subóptima legibilidad lingüísti- 
ca, pudiendo dificultar su comprensión al ciudadano medio y potencial consumidor del medicamento. Por tanto, y mientras no sean corregidas las deficiencias en la escritura de los prospectos, la implicación de todos los profesionales de la salud en la información y resolución de dudas al usuario puede resultar imprescindible para un correcto tratamiento, especialmente respecto a las indicaciones, contraindicaciones y los posibles efectos adversos de los medicamentos: no se debería obviar que la información escrita debe ser un complemento a la comunicación entre el personal sanitario y el paciente.

\section{BIBLIOGRAFÍA}

1. Martínez de Sousa J. Manual de edición y autoedición. Madrid, Pirámide 2005.

2. Asociación Española de Derecho Farmacéutico. La redacción del prospecto: recomendaciones para mejorar su comprensión. Madrid: ASEDEF; 2007.

3. Barrio-Cantalejo IM, Simón-Lorda P, Melguizo M, Escalona I, MariJuán MI, Hernando P. Validación de la Escala INFLESZ para evaluar la legibilidad de los textos dirigidos a pacientes. An Sist Sanit Navar 2008; 31: 135-152.

4. March-Cerdá JC, Prieto-Rodríguez MA, Ruiz-Azarola A, Simón-Lorda P, Barrio-Cantalejo I, Danet A. Mejora de la información sanitaria contenida en los prospectos de los medicamentos: expectativas de pacientes y de profesionales sanitarios. Aten Primaria 2010; 42: 22-27.

5. NutBEAm D. Health literacy as a public health goal: a challenge for contemporary health education and communication strategies into the 21st century. Oxford University Press 2000; 15: 259-267.

6. Palazón Sánchez C, Segura Pérez J, Renedo Villaroya A, Palazón Sánchez El, JC Pardo Talavera $\mathrm{JC}$ et al. Intoxicaciones agudas graves en un servicio de medicina intensiva durante doce años. Rev Esp Salud Pública 2000; 74: 55-63.

7. Medina González L, Fuentes Ferrer ME, Suárez Llanos, JP, Arranz Peña Mi, Ochoa Mangado E. Epidemiología de las intoxicaciones medicamentosas durante un año en el Hospital Universitario Ramón y Cajal Rev Clin Esp. 2008; 208: 432-436.

8. Caballero Vallés PJ, Dorado Pombo S, Díaz Brasero E, García Gill M, Yubero Salgado L, Torres РАсно $\mathrm{N}$ et al. Vigilancia epidemiológica de la intoxicación aguda en el área sur de la comu- nidad de Madrid: estudio Veia 2004. An Med Interna (Madrid) 2008; 25: 62-68.

9. Redacción PRNoticias. Prospectos difícilmente comprensibles. PRNoticias. 23 de noviembre de 2005. Salud. [Online] Acceso el 30/07/2013. Disponible en: http:// www.prnoticias.com/index.php/salud/762prsalud/10076297-prospectos-dificilmentecompresibles.

10. Blanco Pérez A, Gutiérrez Couto U. A propósito de la legibilidad en salud. Sociedad Iberoamericana de Información científica (SIIC), 2004. [Online] Acceso el 10/10/2013. Disponible en: http://www.siicsalud.com/dato/ dat039/04726001.htm

11. IMS Health [sede web]. Danbury (Connecticut): IMS Health; 2013. Acceso 12/08/2013. Disponible en: http://www.imshealth.com/ portal/site/imshealth.

12. Szigriszt Pazos F. Sistemas predictivos de legilibilidad del mensaje escrito: fórmula de perspicuidad [Tesis Doctoral] Madrid: Universidad Complutense de Madrid, 1993.

13. Barrio Cantalejo IM Legibilidad y Salud. Los métodos de medición de la legibilidad y su aplicación al diseño de folletos educativos sobre salud. Tesis doctoral, Universidad Autónoma de Madrid, Facultad de Medicina, 2007.

14. San Norberto EM, Gómez-Alonso D, Trigueros JM, Quiroga J, Gualis J, Vaquero C. Legibilidad de los documentos de consentimiento informado de la SEACV. Angiología 2012; 64: 76-83.

15. Barrio-Cantalejo IM, Simón-Lorda P. ¿Pueden leer los pacientes lo que pretendemos que lean? Un análisis de la legibilidad de materiales escritos de educación para la salud. Aten Primaria 2003; 31: 409-414.

16. Barrio-Cantalejo IM, Simón-Lorda P, March-CerDÁ JC, PrIETo-Rodríguez MA. Legibilidad gramatical de los prospectos de los medicamentos de más consumo y facturación en España en 2005. Rev Esp Salud Pública 2008; 82: 559566.

17. European Commission. Guideline on the readability of the labelling and package leaflet of medicinal products for human use. Brussels: European Commision; 2009.

18. Real Decreto 1345/2007, de 11 de octubre, por el que se regula el procedimiento de autorización, registro y condiciones de dispensación de los medicamentos de uso humano fabricados industrialmente. Boletín Oficial del Estado 267, de 7 de Noviembre de 2007.

19. Real Decreto $1091 / 2010$, de 3 de septiembre, por el que se modifica el Real Decreto $1345 / 2007$, de 11 de octubre, por el que se 
regula el procedimiento de autorización, registro y condiciones de dispensación de los medicamentos de uso humano fabricados industrialmente, y el Real Decreto 1246/2008, de 18 de julio, por el que se regula el procedimiento de autorización, registro y farmacovigilancia de los medicamentos veterinarios fabricados industrialmente. Boletín Oficial del Estado 246 de 12 de Octubre de 2010.

20. Dirección de la Agencia Española de Medicamentos y Productos sanitarios. Criterios de adecuación al RD 1345/2007 en relación con Las consultas a pacientes o usuarios (test de legibilidad), ficha técnica, prospecto paciente y etiquetado. Madrid: Agencia Española de Medicamentos y Productos Sanitarios; 2008.

21. Cardoso P, Curbelo J, Palazzesi A, Álvarez G, Cargnel E. Comprensibilidad de un prospecto de medicamento de venta libre. Rev Argent Salud Pública 2012; 3: 30-35.

22. Lloyd LL, Ammary NJ, Epstein LG, Johnson R, RHEE K. A transdisciplinary approach to improve health literacy and reduce disparities. Health Promot Pract 2006; 7: 331-335.

23. Berkman ND, Sheridan SL, Donahue KE, Halpern DJ, CROTTY K. Low health literacy and health outcomes: an updated systematic review. Ann Intern Med 2011; 155: 97-107.
24. Ministerio de Educación, Cultura y Deporte. Instituto Nacional de Evaluación Educativa. Panorama de la educación indicadores de la OCDE 2013. Informe español. Madrid 2013.

25. GibBons MC. Common ground: Exploring policy approaches to addressing racial disparities from the left and the right. J Health Care Law Policy 2006; 9: 48-76.

26. Smedley BD, Stith AY, Nelson AR, editors. Unequal Treatment: Confronting Racial and Ethnic Disparities in Health Care. Washington, DC: Institute of Medicine 2002.

27. Sentell TL, Halpin HA. Importance of adult literacy in understanding health disparities. $\mathrm{J}$ Gen Intern Med 2006; 21: 862-886.

28. LuRIE N, DuBowitz T. Health disparities and access to health. JAMA 2007; 297: 1118-1121.

29. Barrio-Cantalejo, P. Simón-Lorda, M. Melguizo Jiménez, A. Molina Ruiz C. Consenso sobre los criterios de legibilidad de los folletos de educación para la salud. An Sist Sanit Navar 2011; 34: 153-165.

30. Escudero Carretero MJ, Sánchez Gómez S, González Pérez R, Sanz Amores R, Prieto Rodríguez MA, FERNÁNDEZ DE LA Mota E. Elaboración y validación de un documento informativo sobre adeno-amigdalectomía para pacientes. An Sist Sanit Navar 2013; 36: 21-23. 